\title{
Deterministic HEER Protocol for Heterogeneous Reactive WSNs
}

\author{
Navneet Kaur \\ Research Scholar, Deptt of CSE, \\ D.A.V.I.E.T Jalandhar, \\ Punjab, India.
}

\author{
Manjinder Singh Kahlon \\ Asstt. Professor, Deptt of CSE, \\ D.A.V.I.E.T Jalandhar, \\ Punjab, India.
}

\begin{abstract}
WSNs are playing a crucial role in real world applications. Due to the features of the resource-constrained and battery-aware sensors; in WSNs energy utilization has found to be a major interesting subject of research. WSNs compose batterypowered nodes which are connected with the base station to for certain action or task. As sensor nodes are battery-powered i.e. will become dead after the consumption of the battery which is also called lifetime of WSNs. So using the energy in wellorganized way may result in prolonging the lifetime of the WSNs. This paper has proposed a new HEER protocol which is deterministic in nature instead of randomization i.e. based on the probability. The proposed technique is designed and implemented in MATLAB. The comparison among the proposed and existing HEER has shown that the proposed HEER has better results in terms of network lifetime and stability period.
\end{abstract}

Keywords: Wireless Sensor Network, Threshold-sensitive Stable Election Protocol, Low Energy Adaptive Clustering Hierarchy, Stable Cluster Head Election Protocol, Heterogeneity-aware Hierarchical Stable Election Protocol.

\section{INTRODUCTION}

Technology has advanced to artificial nursing extent such that it moved from wired to wireless domain. The wireless devices are area unit purposeful relying upon their battery life. Wireless communication technologies area unit endlessly growing in various areas and supply new and higher opportunities for various business environments. Recent development in wireless communication and physics have sceptre the readying of tiny, comparatively in-expensive and low-power devices referred to as small sensors which will be connected by a wireless network. These wireless micro sensor networks present a new standard for obtaining data from the surrounding environment and also allow the reliable examining of a number of environments for various applications. Wireless sensor networks have gained so much importance over the past few years because of the many applications in which the sensors can be used to monitor and control different physical phenomenon. WSNs are among the most widely used in adhoc wireless networks. WSNs are referred as infrastructure less networks that are capable of wireless communication. Recent technological advancements have enabled the development of relatively small, inexpensive wireless sensors that consume low power. These sensors can be deployed at a cost much less than those traditional wired systems. Wireless sensor network is a small system that is able to communicate over short distances. This small system is made up of sensor nodes. Sensor nodes are imagined as small computers, extremely basic in terms of their components and interfaces. It is a collection of hundreds and thousands of sensor nodes that are organized into a cooperative network. These nodes are adaptive and self-organized and integrate the functions of collecting data, processing it and then communicating it to the other nodes in the network. These nodes work together to form a powerful sensor network. The sensing elements sense data from the environment, the processing system performs computation on the sensed data and the communication or radio system exchange the information with other nodes in the system. These sensors can detect several environmental events such forest fire, wind velocity, humidity, and soil temperature. This information can then be transmitted to a base station (BS) or sink. To extend the lifetime of the network, efficient use of available energy is the subject to be considered. WSN lifetime is the time interval between the start of the first transmission until the expiry of the last alive node i.e. the time when the operation starts until the percentage of the sensor nodes fall below a specific threshold. Since, most of the energy is consumed due to communication, so an efficient energy saving routing protocol is required to extend the survival rate or the network lifetime. Routing protocols have the capability to reduce the energy consumption of WSNs by choosing minimal routes. Among many routing protocols, the most effective is the cluster-based technique. In cluster-based routing, the sensor nodes are grouped together into a number of clusters and the nodes of the cluster send their data to a specific node called cluster head $(\mathrm{CH})$. The cluster head $(\mathrm{CH})$ is present in each cluster and is responsible for collecting the data from the other nodes. After gathering all the data from sensor nodes, the $\mathrm{CH}$ then transmits it to the sink or Base station (BS) and these local Base Stations transmit the data to the global BS, where it is accessed by the end user. The base stations are one or more components of the WSN with much more communication resources and computational energy. They act as the gateway between sensor nodes and the end user as they typically forward data from the WSN on to a server. The cluster heads are selected based upon a specific criterion. Since $\mathrm{CHs}$ are an important part of cluster-based routing protocols, the $\mathrm{CH}$ selection affects the network lifetime, energy consumption rate and the delay occurred in the delivery of packets.

\section{HEER}

HEER [12] is defined as Hybrid Energy Efficient Reactive Protocol for Wireless Sensor Networks. In HEER the Cluster Head $(\mathrm{CH})$ selection is based on the ratio of residual energy of node and average energy of network. Moreover, to conserve more energy, it introduced the Hard Threshold (HT) and Soft Threshold (ST). Finally, the simulation result shows that the protocol has not only prolonged the network lifetime but also significantly increased stability period. It improves the stable region for clustering hierarchy process for a reactive network in homogeneous and heterogeneous environment. It uses the initial and residual energies of the nodes to become Cluster Head i.e. similar to that of DEEC (Distributed energy efficient clustering protocol) .It does not require any global knowledge 
of energy at any election round. When cluster formation is done, the $\mathrm{CH}$ transmits two threshold values, i.e. $H T$ and $S T$. The nodes sense their environment repeatedly and if a parameter from the attributes set reaches its $H T$ value, the node switches on its transmitter and transmits data.

\section{Important Features:}

1) HEER performs best for time critical applications in both homogeneous and heterogeneous environment.

2) It reduces the number of transmissions resulting in the reduction of energy consumption.

3) It increases the stability period and network lifetime.

\section{RELATED WORK}

Due to small batteries [1] in WSNs, the optimal and efficient usage of energy is an important factor. To enhance the life time of the network, clustering is the most efficient technique. In ECRSEP, the CHs are selected based on the weighted election probabilities of each node according to the Energy Consumption Rate (ECR) of each node. But its limitation is that all nodes should be capable of becoming a $\mathrm{CH}$. Each node in TSEP [2] maintains a routing table which keeps record of the destinations and the number of hops needed to reach the destination. The nodes keep sensing the network for any kind of changes in the system can communicate the data sensed to the other nodes in the network. The reactive routing protocols, unlike the proactive, do not sense the network on a regular basis. The constant updating of routing table is not required. This is on-demand protocol which means that the nodes search for route when needed and then transmit the data. TSEP is a reactive protocol which uses three levels of heterogeneity. These protocols respond instantly to the changes in the parameters of the network. The transmission of data consumes more energy than sensing and takes place only when a certain threshold is achieved. HSEP [3] protocol is heterogeneousaware in the sense that the election probabilities of the nodes to become $\mathrm{CHs}$ are weighted by their initial energy relative to other nodes in the network. It prolongs the time interval before the death of first node in the system thus increasing the stability period. As the distance between the cluster head and sink increases the power consumption is also greatly increased. HSEP reduces the transmission cost from $\mathrm{CH}$ to base station by considering two types of cluster hierarchies: primary and secondary CHs. The new LEACH-N [4] protocol modifies the process of $\mathrm{CH}$ selection by adding the factor of residual energy while calculating $T(n)$ which is

$$
\mathrm{T}(\mathrm{n})=\left\{\begin{array}{c}
\frac{\mathrm{p}}{1-\mathrm{p} \cdot\left(\mathrm{r} \bmod \frac{1}{p}\right)} * \frac{E_{\text {init }}-E_{\text {curngnit }}}{E_{\text {init }}} \\
0, \quad \text { otherwise }
\end{array}, \mathrm{n} \in \mathrm{G}\right.
$$

$E_{\text {init }}$ is the initial energy, $E_{\text {current }}$ is the node's current residual energy. By applying this technique, the nodes who have consumed more energy will have less probability to become $\mathrm{CH}$ again. This ensures optimized way of $\mathrm{CH}$ selection and prolongs the network lifetime. The residual factor thus, reduces the energy cost of network nodes. The $\mathrm{CH}$ [5] node in the network broadcast to the radium $\mathrm{R}$ and forms the measuring area. The nods in the measuring area reflect back the signals to $\mathrm{CH}$. The broadcasting radium $\mathrm{R}$ measures the nearby node distribution. The total measuring area of $\mathrm{CH}$ reaches a cover rate, such that the node distribution in measuring area reflects node distribution in clustering area. Also, as the R increases, the overlap rate also increases. This leads to increase in energy consumption. The node which has low node density if selected the $\mathrm{CH}$, the other nodes need to travel a long distance for communication. The ESEP [7] is a protocol for cluster-head selection in a hierarchical clustered heterogeneous network.
The authors have assumed that the nodes are static and are distributed randomly in the network and the dimensions of the sensor field and sink are known. SEP was based on weighted election probabilities of each node to be a cluster head as per their energies. It considered two types of nodes: advance nodes and normal nodes with advance nodes having high amount of initial energy than the normal nodes of the network. In SCHE [8], the nodes will always have some data to transmit to the base station or sink. It also uses the concept of data aggregation to avoid overload of information or the access to data. It obtains the stable probability to select the $\mathrm{CH}$. An optimum value of the probability is obtained which helps to select a $\mathrm{CH}$. The simulations were performed to evaluate the energy consumption by LEACH and SCHE. For the same transmission distance between the base station and other nodes, the results show that SCHE consumes less energy as compared to LEACH due to stable cluster head election probability whereas LEACH applies random cluster head selection. SEP [9] assumes that the base station is not energy constrained compared to the nodes of the system. Also the nodes are assumed to be uniformly distributed over the field. SEP uses two types of nodes based on their energy level. The nodes that have more energy relative to other nodes are referred as advanced nodes and the other nodes are normal nodes. The clustering hierarchy of LEACH protocol is applied to heterogeneous environment with differing energy levels among the nodes. SEP attempts to maintain the energy consumption of the network by considering advance nodes that have higher energy, to become cluster-heads more often than the normal nodes. By adding a factor of advance nodes, it is observed that the total energy of the system increases by a factor $(1+\alpha) . m$ where $\alpha$ is the additional energy factor between advance and normal nodes and $m$ is the fraction of advance nodes in the system. The stable region could also be increased by that factor if each normal and advance node becomes a cluster-head once each round of an epoch. The simulation results show that the SEP protocol is successful in extending the stable region by assigning probabilities weighted by the initial energies of the nodes. And due to increased stability, the throughput of SEP is also higher than LEACH protocol. TEEN [10] is as reactive routing protocol as it responds immediately to the changes in the value of certain attributes. Each time a cluster changes, the cluster-head broadcasts the parameters to its members. It defines the hard and soft thresholds. Hard threshold is a value beyond which the node sensing this value must turn on its radio and report to its $\mathrm{CH}$. A soft threshold is a small change in the value of sensed attribute that initiates the node to switch on its radio and transmit. The hard threshold tries to reduce the number of transmissions by allowing the nodes to transmit only when the sensed attribute is in the range of interest. The soft threshold reduces the number of transmissions which might have occurred when there is a small change in the value of sensed attribute. TEEN is useful for time-critical applications where the data reaches the user immediately. The energy consumption is reduced as the nodes sense data only when there is a sudden change in the value of attributes. LEACH [11] is a self-organizing protocol that uses randomization to select the cluster base stations to distribute the load evenly among the other nodes in the network. The LEACH protocol selects $\mathrm{CH}$ in two phases: set up phase and steady phase. The nodes organize themselves into local clusters with a cluster-head (CH) node. The high energy cluster-head nodes are randomly rotating among the other sensors so that the battery of just a single sensor is not drained. These $\mathrm{CHs}$ broadcast their status to other sensor nodes and then these nodes decide as to join which of the $\mathrm{CH}$. When all the nodes have chosen their respective clusters, the $\mathrm{CH}$ prepares a schedule for its nodes. 


\section{PROPOSED ALGORITHM}

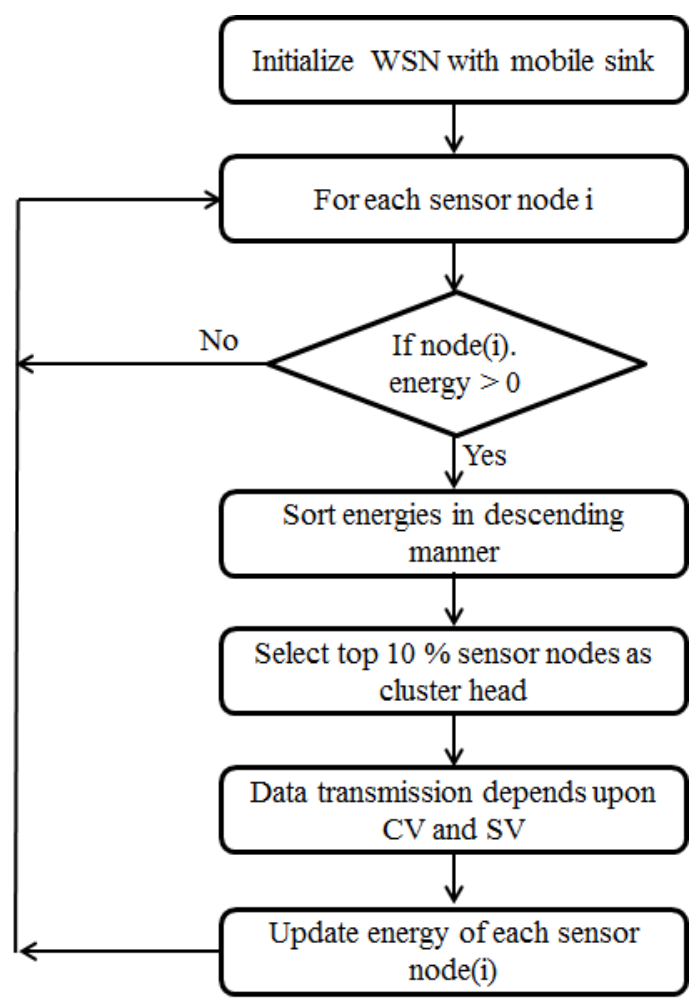

Figure 1: Proposed algorithm

Figure 1 has shown the flowchart of the proposed algorithm. Wireless Sensor Networks (WSNs) are composed of multiple unattended ultra-small, limited-power sensor nodes. These nodes have limited processing, wireless communication and power resource capabilities, which send sensed data to sink or Base Station (BS). A novel technique as efficient Cluster head Replacement has been proposed i.e. HEER. In this the Cluster Head $(\mathrm{CH})$ selection is based on the ratio of residual energy of node and average energy of network. Moreover, to conserve more energy, HEER has also utilized the Hard Threshold (HT) and Soft Threshold (ST). But HEER does not use the inter cluster data aggregation, but it is not deterministic in nature, as cluster head selection is based upon the weighted probabilities. So to overcome this problem a deterministic approach is used to enhance the cluster head selection. The idea behind deterministic cluster head selection is simple; firstly all the nodes will be sorted based upon their residual energies top 10\% nodes will be selected as cluster head. So the selection criteria will have deterministic decisions because node with highest first energy will become cluster head than the probability. It will increase the overall network lifetime.

Following are the various steps required to successfully simulate the proposed technique.

Step1: First of all initialization of the sensor network will be done by setting up various constants and variables of the network.

Step2: For every sensor node $\mathrm{i}$ if any node has 0 or negative energy then node will be set as dead and continue for next step.

Step3: Sort the nodes according to their energies in descending fashion. Select top $10 \%$ nodes as cluster head.

a) Sort the nodes according to their residual energies in descending fashion. b) Select the first $10 \%$ nodes as cluster heads.

Step4: Use SV and CV to transmit data between BS to $\mathrm{CH}$.

Step5: Evaluate energy dissipated and update the remaining energy of each node (i) and move to step 2 again.

\section{EXPERIMENTAL SETUP}

This section contains the experimental setup which has been used in this research paper. Table 1 has shown various constants and variables required to simulate this work. These parameters are standard values used as benchmark for WSNs.

Table 1. Experimental Setup

\begin{tabular}{|l|l|}
\hline Parameter & Value \\
\hline Area(x,y) & 100,100 \\
\hline Base station(x,y) & 50,50 \\
\hline Nodes(n) & 100 \\
\hline Probability(p) & 0.1 \\
\hline Initial Energy(Eo) & 0.5 \\
\hline transmiter_energy & $50 \mathrm{~nJ} / \mathrm{bit}$ \\
\hline receiver_energy & $50 \mathrm{~nJ} / \mathrm{bit}$ \\
\hline Free space(amplifier) & $10 \mathrm{nj} / \mathrm{bit} / \mathrm{m} 2$ \\
\hline Multipath(amplifier) & $0.0013 \mathrm{pJ} / \mathrm{bit} / \mathrm{m} 4$ \\
\hline $\begin{array}{l}\text { a (energy factor between normal and } \\
\text { advance nodes) }\end{array}$ & 1 \\
\hline Maximum lifetime & 7500 \\
\hline Message size & $4000 \mathrm{bits}$ \\
\hline m (fraction of advanced nodes) & 0.1 \\
\hline Effective Data aggregation & $5 \mathrm{~nJ} / \mathrm{bit} / \mathrm{signal}$ \\
\hline
\end{tabular}

\section{PERFORMANCE ANALYSIS}

The implementation of the proposed and the existing HEER has been done in MATLAB using data analysis toolbox, following results has been achieved.

Figure 2 has shown the first node dead time comparison among the proposed and the existing HEER. it has been clearly shown that the first node dead time is more i.e. (2072- HEER and 2306- Proposed HEER) in case of the proposed HEER therefore it outperforms over the available techniques.

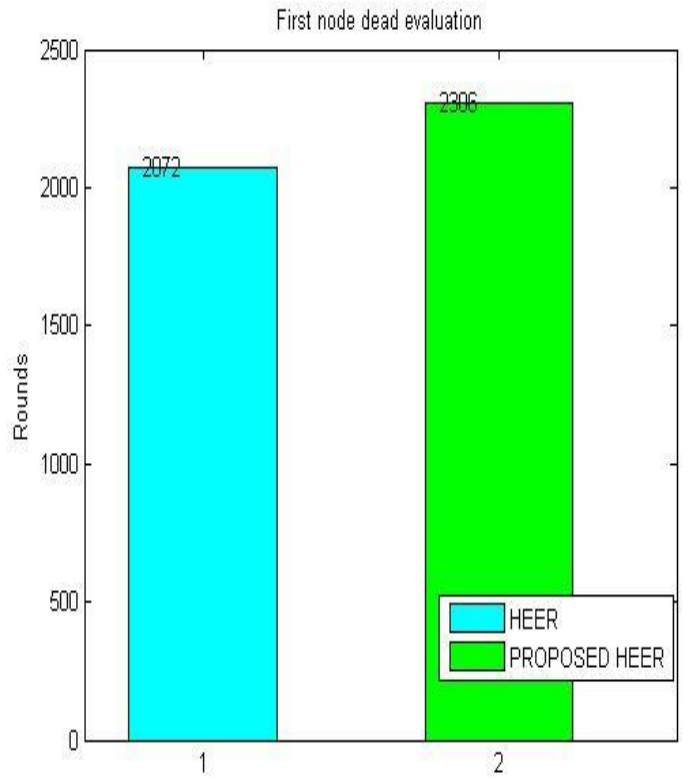

Figure 2: First node dead time evaluation 
Figure 3 has exposed the half node dead time comparison among the proposed and the existing HEER. It has been clearly shown that the half node dead time (2329- HEER and 2494Proposed HEER) is more in case of the proposed HEER therefore it outperforms over the available techniques.

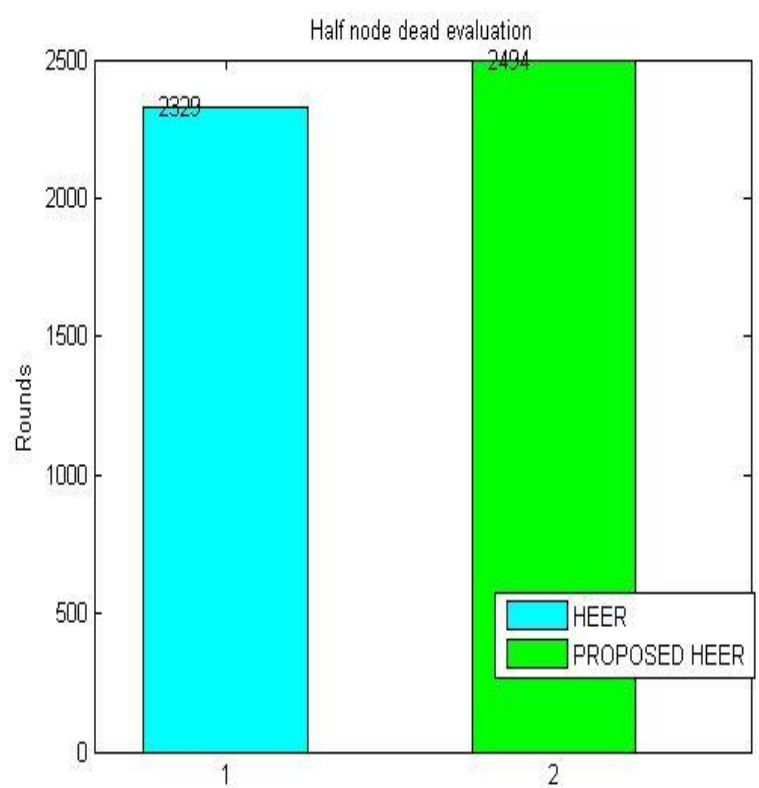

Figure 3: Half node dead time evaluation

Figure 4 has exposed the last node dead time comparison among the proposed and the existing HEER. It has been clearly shown that the last node dead time (3828- HEER and 4926Proposed HEER) is more in case of the proposed HEER therefore it outperforms over the available techniques.

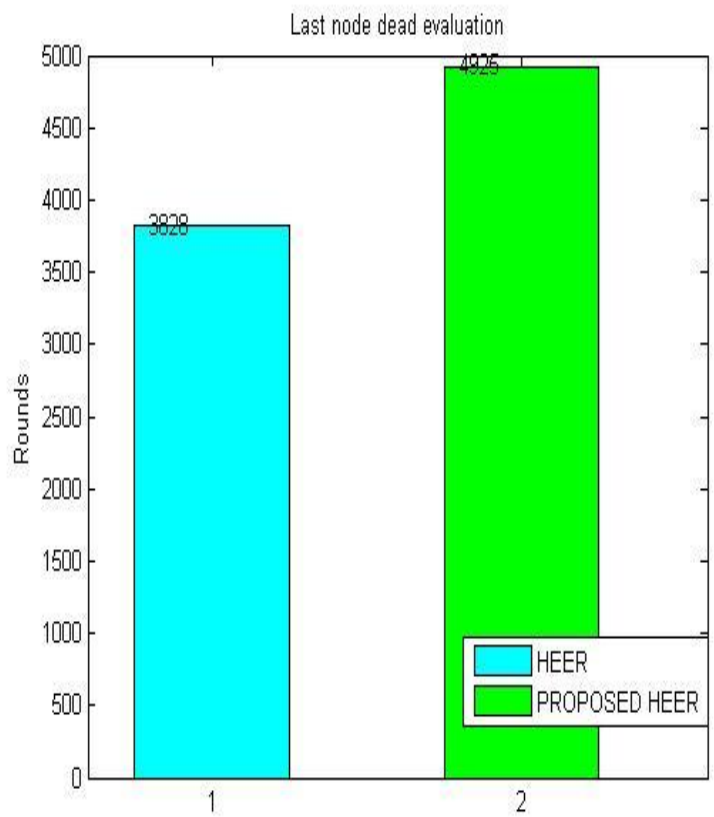

Figure 4: Last node dead time evaluation
Figure 5 has shown the Dead nodes comparison among the proposed and the existing HEER. it has been clearly shown that the dead nodes time has been extended in case of the proposed HEER therefore it outperforms over the available techniques.

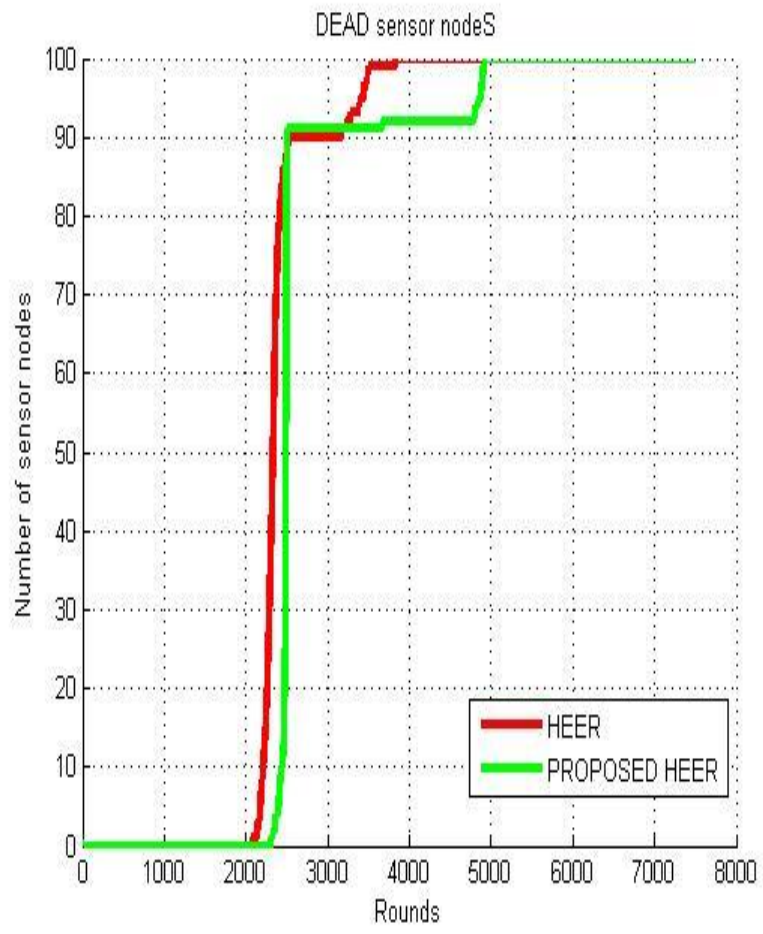

Figure 5: Network lifetime evaluation

Figure 6 has shown the average energy consumption in comparison among the proposed and the existing HEER. It has been clearly shown that the average energy consumption is more in case of the proposed HEER therefore it outperforms over the available techniques.

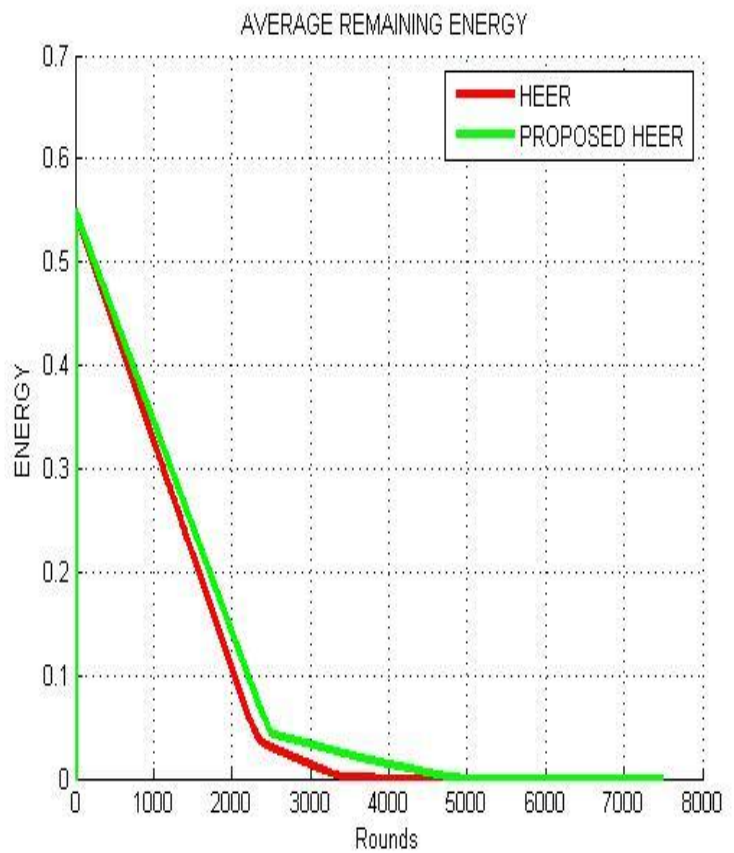

Figure 6: Average Energy Consumption evaluation 
Figure 7 has shown the packets sent to $\mathrm{CH}$ comparison among the proposed and the existing HEER. it has been clearly shown that the packets sent to $\mathrm{CH}$ is more in case of the proposed HEER therefore it outperforms over the available techniques.

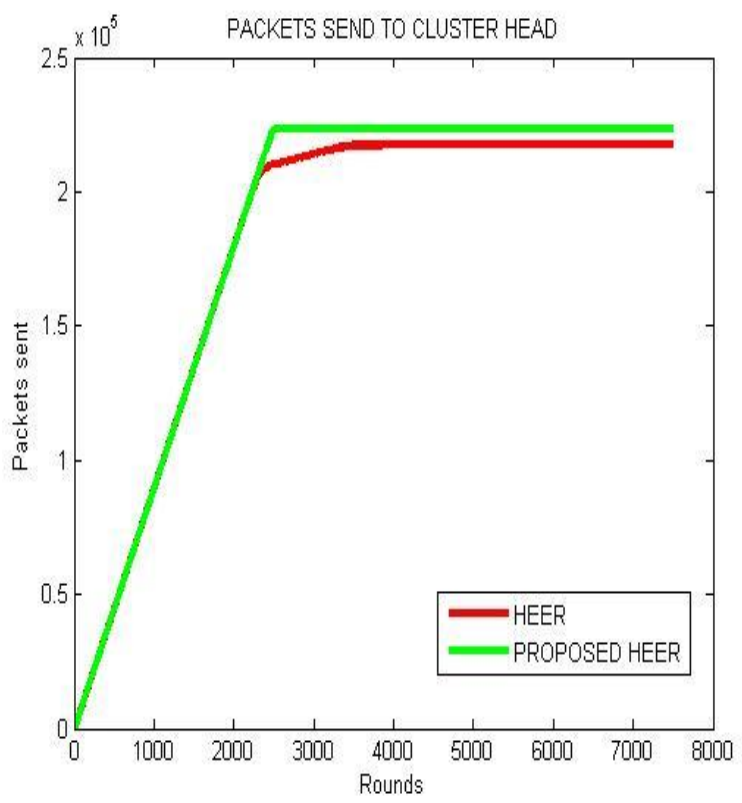

Figure 7: Packets sent to $\mathrm{CH}$ evaluation

Figure 8 has shown the packets sent to BS comparison among the proposed and the existing HEER. It has been clearly shown that the packets sent to BS are more in case of the proposed HEER therefore it outperforms over the available techniques.

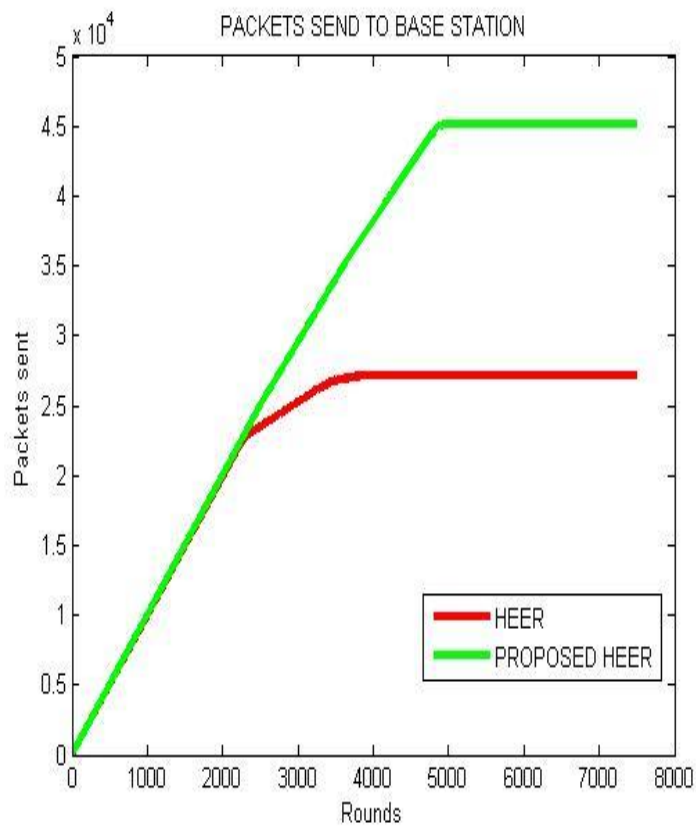

Figure 8: Packets sent to BS evaluation

Figure 9 has shown the improvement of the proposed HEER in (\%) for network stable period i.e. $15.29 \%$ and the network lifetime $29.023 \%$.

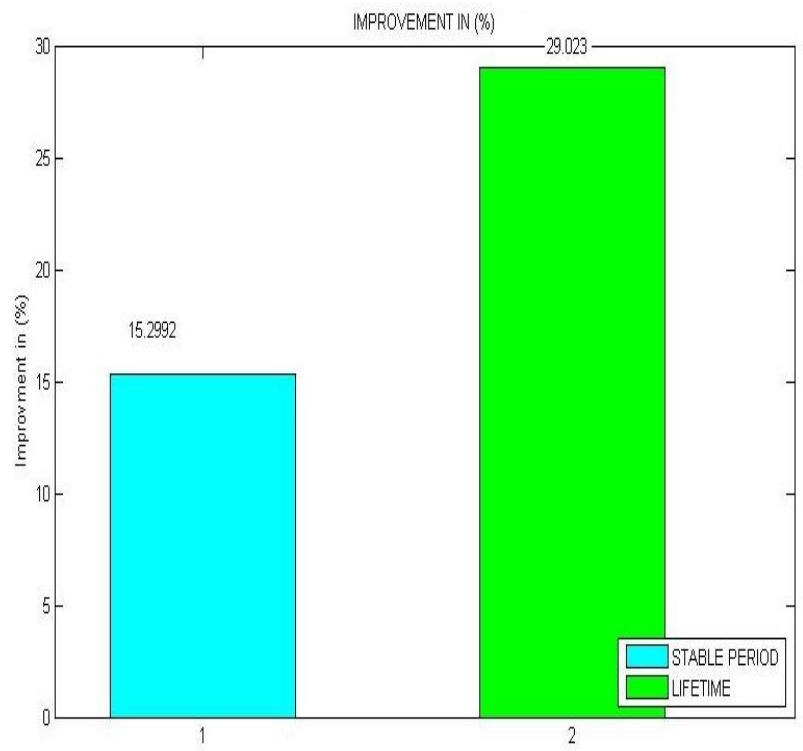

Figure 9: Improvement of proposed HEER

\section{CONCLUSION AND FUTURE WORK}

Wireless Sensor Networks (WSNs) are composed of multiple unattended ultra-small, limited-power sensor nodes. These nodes have limited processing, power resource capabilities and wireless communication, which send sensed data to sink or Base Station (BS). A novel technique as efficient Cluster head Replacement has been proposed i.e. HEER. In this the Cluster Head $(\mathrm{CH})$ selection is based on the ratio of residual energy of node and average energy of network. Moreover, to conserve more energy, HEER has also utilized the Hard Threshold (HT) and Soft Threshold (ST). But HEER does not use the inter cluster data aggregation, but it is not deterministic in nature, as cluster head selection is based upon the weighted probabilities. So to overcome this problem a deterministic approach has been proposed to enhance the cluster head selection. The idea behind deterministic cluster head selection is simple; firstly all the nodes will be sorted based upon their residual energies top $10 \%$ nodes will be selected as cluster head. So the selection criteria have deterministic decisions because node with highest first energy will become cluster head than the probability. The comparative analysis has shown that the proposed HEER outperforms over the HEER protocol with respect to the stable period and the network lifetime. In near future the further enhancement will be done by using the data aggregation techniques.

\section{REFERENCES}

[1] Rehman, O., Javaid, N., Manzoor, B., Hafeez, A., Iqbal, A., \&Ishfaq, M. (2013). Energy Consumption Rate based Stable Election Protocol (ECRSEP) for WSNs. Procedia Computer Science, 19, 932-937.

[2] Kashaf, A., Javaid, N., Khan, Z. A., \& Khan, I. A. (2012, December). TSEP: Threshold-sensitive Stable Election Protocol for WSNs. In Frontiers of Information Technology (FIT), 2012 10th International Conference on (pp. 164-168). IEEE.

[3] Khan, A. A., Javaid, N., Qasim, U., Lu, Z., \& Khan, Z. A. (2012). HSEP: Heterogeneity-aware Hierarchical Stable Election Protocol for WSNs. arXiv preprint arXiv:1208.2335. 
[4] Li, Yuling, Luwei Ding, and Feng Liu. "The improvement of LEACH protocol in WSN." Computer Science and Network Technology (ICCSNT), 2011 International Conference on. Vol. 2. IEEE, 2011.

[5] Peng, J., Chengdong, W., Yunzhou, Z., \&Fei, C. (2011, September). A Low-Energy Adaptive Clustering Routing Protocol of Wireless Sensor Networks. InWireless Communications, Networking and Mobile Computing (WiCOM), 2011 7th International Conference on (pp. 14). IEEE

[6] Aderohunmu, Femi A., and Jeremiah D. Deng. "An Enhanced Stable Election Protocol (and Heterogeneous WSN." XH Wu, S. Wang," Performance comparison of LEACH on LEACH-C protocols by NS2," Proceedings of 9th International Symposium SEP) for Clustered Distributed Computing and Applications to Business, Engineering and Science. Hong Kong, China. 2010.

[7] Islam, M. M., Matin, M. A., \&Mondol, T. K. (2012, June). Extended Stable Election Protocol (SEP) for threelevel hierarchical clustered heterogeneous WSN. In Wireless Sensor Systems (WSS 2012), IET Conference on (pp. 1-4). IET.
[8] Muhamad, W. N. W., Dimyati, K., Mohamad, R., Haron, M. A., Sarnin, S., Wahab, N., \& Aziz, N. H. A. (2008, December). Evaluation of Stable Cluster Head Election (SCHE) routing protocol for wireless sensor networks. In RF and Microwave Conference, 2008. RFM 2008. IEEE International (pp. 101-105). IEEE.

[9] Smaragdakis, Georgios, Ibrahim Matta, and AzerBestavros. SEP: A stable election protocol for clustered heterogeneous wireless sensor networks. Boston University Computer Science Department, 2004.

[10] Manjeshwar, Arati, and Dharma P. Agrawal. "TEEN: ARouting Protocol for Enhanced Efficiency in Wireless Sensor Networks." IPDPS. Vol. 1. 2001.

[11] Heinzelman, Wendi Rabiner, AnanthaChandrakasan, and HariBalakrishnan. "Energy-efficient communication protocol for wireless microsensor networks." System Sciences, 2000. Proceedings of the 33rd Annual Hawaii International Conference on. IEEE, 2000.

[12] N. Javaid, S. N. Mohammad, K. Latif, U. Qasim, Z. A. Khan, M. A. Khan."HEER; Hybrid energy efficient reactive protocol” IEEE, August 2013. 